\title{
Article \\ Low-altitude atmospheric turbulence sounding on the basis of unmanned aerial vehicle
}

\author{
Alexander Shelekhov ${ }^{1, *}$, Aleksey Afanasiev ${ }^{2}$, Alexey Kobzev ${ }^{1}$, Evgenia Shelekhova ${ }^{1}$, Alexey \\ Tel'minov $^{1}$, Alexander Molchunov ${ }^{1}$ and Olga Poplevina ${ }^{1}$ \\ 1 Institute of Monitoring of Climatic and Ecological Systems SB RAS, 10/3, Academichesky Ave, 634055, \\ Tomsk \\ 2 V.E. Zuev Institute of Atmospheric Optics SB RAS, 634055, Tomsk, Russia, 1, Academician Zuev square \\ * Correspondence: ash@imces.ru; Tel.: +7-952-883-9923
}

\begin{abstract}
Based on the theory of turbulence, equations are derived for estimations of turbulent fluctuations of the longitudinal and transverse components of the wind velocity during ideal hovering of a quadcopter in a turbulent atmosphere. We present the results of experiments which were carried out on the territory of the Geophysical Observatory of Institute of Monitoring of Climatic and Ecological Systems, Siberian Branch, Russian Academy of Sciences, located in Tomsk Akademgorodok on the territory with complex orography, in a parkland zone with buildings of research institutes and motorways. Time series of turbulent fluctuations of the longitudinal and transverse components of wind velocity fluctuations were received with the use of an automated weather station, and time series of estimates of these components, from data of a DJI Phantom 4 Pro quadcopter during hovering. According to the automated weather station data, anisotropy was observed in one experiment during measurements in the atmosphere, but this phenomenon was not observed in the other experiment: the fluctuation spectra of all components of wind speed fluctuations coincide. The spectra of fluctuations of the longitudinal and transverse wind velocity components based on the automated weather station data and UAV telemetry are presented. The fluctuation spectra of these components for the automated weather station data and quadcopter generally coincide. The behavior of the spectra coincides with the spectrum which corresponds to Kolmogorov-Obukhov " $-5 / 3$ " law within the inertial range. The turbulent spectra of the wind velocity fluctuations obtained with the use of the automatic weather station and with the unmanned aerial vehicle differ in the high-frequency spectral region.
\end{abstract}

Keywords: unmanned aerial vehicle; low-altitude sounding; atmospheric turbulence; wind velocity; fluctuations; spectrum

\section{Introduction}

Low-altitude sounding of atmospheric turbulence is of great importance for many scientific and applied problems. Traffic control of unmanned aerial vehicles (UAV) of a "smart city", study of changes in the urban climate, monitoring of air pollution in urban environment and other regions with complex orography are among problems which could be solved in the presence of reliable high-resolution data on the state of atmospheric turbulence. The use of UAV, both fixed-wing and rotary-wing, is one of the main directions of development of the low-altitude sounding of turbulent flows in the atmosphere. The paper [1] presents the historic review and describes promises of a system of atmospheric observations. In addition, this paper discusses the widely used specialized observation systems, such as radars, lidars, and research aircraft, and presents some examples of their application for weather forecasting and prediction of climate.

The analysis given in [2-4] showed multi-rotor UAS to be more promising instrument for remote low-altitude sounding than observation balloons with sensors, since the former are cheap, easy to operate, longeval in a wide range of atmospheric conditions, and reusable. The advantages of multirotor UAS include vertical take-off and landing, as well as the capability of hovering at a fixed point. These advantages make this UAS type an ideal platform for measuring vertical profiles or 
temporal variations in the atmosphere. The results of the workshop on the problem of providing weather information, including data on atmospheric turbulence, for UAV and its integration into Unmanned Traffic Management are summarized in [5]. According to the numerical simulation results [6], constant wind, turbulent flow, many types of wind shear, and propeller vortex strongly affect YAV at low altitudes. In this work, a turbulence was simulated with Dryden and Von Karman models. Recent developments of the unmanned aerial vehicles (UAVs) opens wide opportunities for their application for in-situ meteorological measurements in the atmospheric boundary layer [7].

Results of remote sounding of atmospheric turbulence with the use of a small unmanned meteorological observer (SUMO), which is a fixed-wing, are described in [8-10]. The SUMO is equipped with a miniaturized probe for measuring atmospheric turbulence near wind farms with a time resolution of $100 \mathrm{~Hz}$. Measurements of the spectra of fluctuations of the crosswind and the vertical wind are presented. The study has demonstrated a capability to measure the wind vector with an accuracy comparable to well-established radiosoundings.

Fast sensors for sensing turbulence in the atmospheric boundary layer were designed in [11] for fixed-wing of $18-28 \mathrm{~kg}$ in weight. The fluctuation spectra of the vertical and horizontal wind components, measured by such sensors fixed at a UAV and with an acoustic anemometer, were recorded during tests. The marine atmospheric boundary layer and ocean surface are comprehensively studied in [12] with the help of fixed-wing UAV and other instruments. The high resolution results of the study of the fine turbulence structure with the help of DataHawk UAV and comparison with numerical simulation results are given in [13].

According to [14,15], a turbulent 3D wind vector can be measured with a fixed-wing UAV with a five-hole probe and an inertial navigation system. Such measurements were performed in the atmospheric boundary layer and near wind turbines. The measured profiles of the wind speed and direction and of the kinetic energy of turbulence are presented in [15].

Results of wind profiling with the help of an acoustic anemometer fixed at a hexacopter $1 \mathrm{~m}$ wide are given in [16]. Wind speed and direction measured are in a good agreement with observations made by instruments mounted at a meteorological mast $55 \mathrm{~m}$ high in a wide range of wind velocities.

In [2], direct and indirect techniques were used to determine the wind speed and direction. In the direct technique, a 2D acoustic anemometer was mounted at a high carrying capacity hexacopter, and in the indirect technique, the wind speed and direction were estimated from the data on the pitch and roll angles. The data on the angles were recorded at a frequency of $10 \mathrm{~Hz}$, and data on the wind speed and direction were recorded at a frequency of $1 \mathrm{~Hz}$ by anemometers mounted at three $10-\mathrm{m}$ towers at the experimental site. The study has shown that direct and indirect techniques allow estimating the wind speed with equal accuracy, while wind direction is more accurately estimated by the indirect technique. The indirect technique was earlier suggested in [17], but it was tested in a wind tunnel, but not in the field conditions like in [2].

The studies [3-4] were devoted to the development of an approach to estimation of the vertical profile and horizontal wind velocity components with the use of a quadcopter in hover and vertical flight. The technique suggested for measuring the horizontal wind was validated using two sodars and an acoustic anemometer. The time resolution of the sodars was 30 and $300 \mathrm{~s}$, and of the anemometer, $1 \mathrm{~s}$. The study results [3-4] show the general efficiency of the technique for measuring the vertical wind profile in the lower atmosphere using a quadcopter.

Wind velocity estimates in a turbulent atmosphere with the use of UAV without wind sensors and the machine learning approach are analyzed in [18]. The Dryden model and the LES model of the atmospheric boundary layer are used in the simulation. The results show that the use of a neural network with long short-term memory provides for better estimates than linear approaches, especially under strong turbulence.

The K Nearest Neighborhood learning-based method is suggested in [19] for rotary-wing vertical take-off and landing UAVs. Experimental results obtained with the use of a Parrot AR.Drone demonstrate the accuracy and robustness of the developed wind estimation algorithms under hovering conditions. 
The technique for measuring an atmospheric turbulence and temperature at a space point on the basis of integration of fast-response multihole pressure probe and a thermocouple fixed at a multirotor UAV-based platform is described in [20]. The technique allows accurate measurement of time series of the three components of the velocity vector and temperature at any space point where the UAV can fly within the limits of its autonomy.

A method of measuring wind speed using the data logged by the UAV is proposed in [21]. Experiments were conducted at a test site with laminar wind conditions with the UAV hovering next to a static 2D ultrasonic anemometer. During the flight tests, the wind velocity varied within $0-5$ $\mathrm{m} / \mathrm{s}$. Horizontal wind estimates provide good results with root mean square error (RMSE) values between $0.26-0.29 \mathrm{~m} / \mathrm{s}$ for wind speed, as well as between $4.1-4.9$ for wind direction.

In [22], the air temperature was measured by attaching a temperature sensor $40 \mathrm{~cm}$ above the rotary-wing of the UAV and the UAV-based wind velocity was estimated using attitude data (pitch, roll, and yaw angles) recorded using the UAV's inertial measurement unit. The wind velocity was close to that observed by the automatic weather system within an error of approximately $10 \%$.

The aim of this work is to carry out the comparative analysis of the spectra of fluctuations of the longitudinal and transverse components of wind velocity, measured with an AMK-03 automated weather station (AMK-03 is a 3D acoustic anemometer) [23,24]and a DJI Phantom 4 Pro quadcopter in hovering mode. The experiments were carried out in an urban area with a complex orography on the basis of the Geophysical Observatory of the IMCES SB RAS. The main equations of the turbulence theory are given in the next section, which are required for the correct statement of the problem of low-altitude sounding of atmospheric turbulence using quadcopter without wind sensor. The third and fourth section the equations of estimates of the wind velocity components and estimates of longitudinal and transverse components are provided for quadcopter without wind sensor. The general information about the experiment and the measurement results obtained with the DJI Phantom 4 Pro quadcopter and the AMK-03 automatic meteorological complex are presented in the 5th and 6th sections. The fluctuation spectra of the longitudinal and transverse components in a turbulent atmosphere using the AMK-03 data and quadcopter telemetry are compared.

\section{Model of atmospheric turbulence}

Airflow in the atmosphere is accompanied by random temporal and spatial fluctuations. Therefore, the turbulent air flow velocity field is represented as the sum of the mean and fluctuation components [25,26]:

$$
\boldsymbol{w}(\boldsymbol{r}, t)=\langle\boldsymbol{w}(\boldsymbol{r}, t)\rangle+\boldsymbol{w}^{\prime}(\boldsymbol{r}, t),
$$

where $\langle\boldsymbol{w}(\boldsymbol{r}, t)\rangle$ is the average wind velocity; $\boldsymbol{w}^{\prime}(\boldsymbol{r}, t)$ are the wind velocity fluctuations; $\langle\ldots\rangle$ is the operator of statistical averaging.

In the coordinates commonly accepted in meteorology [26], the $X$ axis is eastward directed (E), the $\mathrm{Y}$ axis is northward directed $(\mathrm{N})$, and the $\mathrm{Z}$ axis is upward directed and is normal to the actual horizon surface. When mathematically describing the wind field fluctuations, the second-rank correlation tensor

$$
B_{p q}\left(\boldsymbol{r}-\boldsymbol{r}^{\prime}\right)=\left\langle w_{p}^{\prime}(\boldsymbol{r}, t) w_{q}^{\prime}\left(\boldsymbol{r}^{\prime}, t\right)\right\rangle
$$

is introduced, where $p$ and $q$ enumerate the orthogonal components of the vector of velocity field fluctuations $\boldsymbol{w}^{\prime}(\boldsymbol{r}, t)$ along the $\mathrm{X}, \mathrm{Y}$, and $\mathrm{Z}$ axes, i.e., $p, q \in\{\mathrm{X}, \mathrm{Y}, \mathrm{Z}\}$. For isotropic turbulence, the correlation tensor is expressed via the longitudinal $B_{r r}(r)$ and transverse $B_{t t}(r)$ components:

$$
B_{p q}(r)=B_{t t}(r)\left(\delta_{p q}-n_{p} n_{q}\right)+B_{r r}(r) n_{p} n_{q}
$$

Where

$$
B_{r r}(r)=n_{p} n_{q} B_{p q}(r)
$$




$$
B_{t t}(r)=\frac{1}{2}\left(\delta_{p q}-n_{p} n_{q}\right) B_{p q}(r) ;
$$

$\boldsymbol{n}=\left\{n_{q}\right\}=\frac{\boldsymbol{r}}{r}, r=|\boldsymbol{r}|$. Summation in Eqs (4) and (5) is carried over double subscripts.

As is well known, horizontal air mass transfer often prevails over vertical motion in the atmosphere; therefore, the behavior of the correlation tensor is studied with respect to the mean horizontal wind velocity direction $\left\langle\boldsymbol{w}_{\|}(\boldsymbol{r}, t)\right\rangle$. In this case, the vector $\mathbf{n}$ in Eqs. (3)-(5) has the form

$$
\boldsymbol{n}=\left\{n_{X}, n_{Y}, 0\right\}=\left\{\frac{\left\langle w_{X}\right\rangle}{\left\langle w_{\|}\right\rangle}, \frac{\left\langle w_{Y}\right\rangle}{\left\langle w_{\|}\right\rangle}, 0\right\},
$$

where $\left\langle w_{X}\right\rangle$ and $\left\langle w_{Y}\right\rangle$ are the mean wind velocity along the $X$ and $Y$ axes; $w_{\|}=\sqrt{\left\langle w_{X}\right\rangle^{2}+\left\langle w_{Y}\right\rangle^{2}}$ is the mean speed of the horizontal turbulent flow.

Substitution of Eq. (6) in longitudinal and transverse correlation tensor components (4) and (5) results in the equations

$$
\begin{gathered}
B_{r r}(r)=\left\langle w_{r}^{\prime}\left(\boldsymbol{r}_{1}, t\right) w_{r}^{\prime}\left(\boldsymbol{r}_{1}+\boldsymbol{r}, t\right)\right\rangle, \\
B_{t t}(r)=\left\langle w_{t}^{\prime}\left(\boldsymbol{r}_{1}, t\right) w_{t}^{\prime}\left(\boldsymbol{r}_{1}+\boldsymbol{r}, t\right)\right\rangle+\left\langle w_{\perp}^{\prime}\left(\boldsymbol{r}_{1}, t\right) w_{\perp}^{\prime}\left(\boldsymbol{r}_{1}+\boldsymbol{r}, t\right)\right\rangle .
\end{gathered}
$$

Here,

$$
w_{r}^{\prime}(\boldsymbol{r}, t)=n_{X} w_{X}^{\prime}(\boldsymbol{r}, t)+n_{Y} w_{Y}^{\prime}(\boldsymbol{r}, t)
$$

is the longitudinal component of the wind velocity fluctuations and

$$
\begin{gathered}
w_{t}^{\prime}(\boldsymbol{r}, t)=-n_{Y} w_{X}^{\prime}(\boldsymbol{r}, t)+n_{X} w_{Y}^{\prime}(\boldsymbol{r}, t), \\
w_{\perp}^{\prime}(\boldsymbol{r}, t)=w_{Z}^{\prime}(\boldsymbol{r}, t)
\end{gathered}
$$

is its transverse components. Equations (9) and (10) show the longitudinal $w_{r}^{\prime}(\boldsymbol{r}, t)$ and transverse $w_{t}^{\prime}(\boldsymbol{r}, t)$ components to be in the horizontal plane XoY; hence, they are horizontal components of the wind velocity fluctuations. The second transverse component of the wind velocity fluctuations $w_{\perp}^{\prime}(\boldsymbol{r}, t)$ is the vertical component of this wind velocity, as is seen from Eq. (11), i.e., it is directed along the $\mathrm{Z}$ axis, which is normal to the XoY plane.

For isotropic fluctuations of the velocity field, the spectral tensor $\Phi_{i j}(k)$ is defined as a scalar function of the wave number $k=|\boldsymbol{k}|$ as

$$
\Phi_{i j}(k)=\frac{1}{4 \pi}\left(\delta_{i j}-\frac{k_{i} k_{j}}{k^{2}}\right) F(k),
$$

where $F(k)=k^{2} E(k)$ is the spectral function; $E(k)$ is the spectral density of the average kinetic energy of a unit air mass [25]; $k_{i}$ are the coordinates of the vector $\boldsymbol{k} ; \delta_{i j}$ is the Kronecker delta.

For isotropic fluctuations, the Kolmogorov-Obukhov "-5/3" law [25]:

$$
E(k) \sim \varepsilon \cdot k^{-5 / 3}
$$

is fulfilled in the inertial range of wavenumbers, where $\varepsilon$ is the dissipation rate of turbulent kinetic energy.

The correlation between the spatiotemporal and spatial parameters of the wind field can be derived on the basis of the Taylor hypothesis about "frozen" turbulent fluctuations [25], the essence of which reduces to the fact that the spatial pattern $\boldsymbol{w}^{\prime}(\boldsymbol{r}, t)$ moves in time with the mean wind velocity $\langle\boldsymbol{w}\rangle$, i.e.,

$$
\boldsymbol{w}^{\prime}\left(\boldsymbol{r}, t+t^{\prime}\right)=\boldsymbol{w}^{\prime}\left(\boldsymbol{r}-\langle\boldsymbol{w}\rangle t^{\prime}, t\right)
$$

The equation which connects the frequency spectrum $\Phi(f)$ of a random field with its spatial spectrum $F(k)$ was derived in [25]. Using this equation, one can write the equation for the frequency spectrum in the case of the Kolmogorov-Obukhov "-5/3" law:

$$
\Phi(f) \sim f^{-5 / 3} .
$$


Equation (15) shows that the frequency spectrum of a random wind velocity damps proportional to the frequency in the power $-5 / 3$.

\section{Estimates of the wind velocity components}

Dynamic equations for the center of gravity of a quadcopter can be written in the inertial coordinates associated with the Earth as [27-30]

$$
\begin{gathered}
\ddot{x}=\left(s_{\phi} s_{\psi}+c_{\phi} s_{\theta} c_{\psi}\right) \frac{T}{m}+\frac{F_{x}}{m^{\prime}} \\
\ddot{y}=\left(-s_{\phi} c_{\psi}+c_{\phi} s_{\theta} s_{\psi}\right) \frac{T}{m}+\frac{F_{y}}{m^{\prime}} \\
\ddot{z}=c_{\phi} c_{\theta} \frac{T}{m}-g+\frac{F_{z}}{m^{\prime}}
\end{gathered}
$$

where $s_{(\bullet)}=\sin (\bullet), c_{(\bullet)}=\cos (\bullet) ; \phi$ is the roll angle; $\theta$ is the pitch angle; $\psi$ is the yaw angle; $T$ is the aerodynamic force generated by propellers; $F_{x}, F_{y}$, and $F_{z}$ are the drag force components along the $\mathrm{x}, \mathrm{y}$, and $\mathrm{z}$ axes; $m$ is the quadcopter mass; $g$ is the gravity acceleration.

The components of the drag force along the $\mathrm{x}, \mathrm{y}$, and $\mathrm{z}$ axes, which arises during a quadcopter flight, have the form

$$
F_{j}=-c_{j}\left(v_{j}-w_{j}\right)
$$

in the linear case and

$$
F_{j}=-\frac{1}{2} \rho C_{j} A_{j} \operatorname{sgn}\left(v_{j}-w_{j}\right) \times\left(v_{j}-w_{j}\right)^{2}
$$

in the square-law case. In Eqs. (20) and (21), $c_{j}$ and $C_{j}$ are the drag coefficient along the $\mathrm{x}, \mathrm{y}$, and $\mathrm{z}$ axes; $j$ is the subscript for enumeration of the orthogonal components of vectors, i.e., $j \in\{x, y, z\} ; v_{j}$ are the quadcopter speed component; $w_{j}$ are the wind velocity components in the atmosphere; $\rho$ is the air density; $A_{j}$ are the projections of the quadcopter area on the corresponding axes; $\operatorname{sgn}(\bullet)$ is the sign function.

As is well known, the ideal hover is possible when $v_{j}=0$ and all forces which act on a quadcopter are compensated. The roll, pitch, and yaw angles in a turbulent atmosphere are sums of the average and fluctuation components: $\phi=\langle\phi\rangle+\phi^{\prime}, \theta=\langle\theta\rangle+\theta^{\prime}$ и $\psi=\langle\psi\rangle+\psi^{\prime}$. Equations (16)-(19) can be transformed to the case of ideal hover by means of their linearization. In the small-angle approximation $\phi, \theta \ll \pi$, at $\psi^{\prime} \ll \pi$ and under the conditions $\ddot{x}=\ddot{y}=\ddot{z}=0$ and $v_{j}=$ 0 , the equations for estimates of the horizontal components of the wind field $\widehat{w}_{x}=w_{x}$ and $\widehat{w}_{y}=w_{y}$ take the forms

$$
\begin{gathered}
\widehat{w}_{x}=-\frac{m g}{c_{x}}\left(\langle\varphi\rangle s_{\langle\psi\rangle}+\langle\theta\rangle c_{\langle\psi\rangle}\right)-\frac{m g}{c_{x}}\left(\varphi^{\prime} s_{\langle\psi\rangle}+\theta^{\prime} c_{\langle\psi\rangle}\right), \\
\widehat{w}_{y}=-\frac{m g}{c_{y}}\left(-\langle\varphi\rangle c_{\langle\psi\rangle}+\langle\theta\rangle s_{\langle\psi\rangle}\right)-\frac{m g}{c_{y}}\left(-\varphi^{\prime} c_{\langle\psi\rangle}+\theta^{\prime} s_{\langle\psi\rangle}\right)
\end{gathered}
$$

in the linear case and

$$
\begin{gathered}
\widehat{w}_{x}=-\operatorname{sgn}\left(\langle\varphi\rangle s_{\langle\psi\rangle}+\langle\theta\rangle c_{\langle\psi\rangle}\right) \sqrt{\frac{2 m g}{\rho C_{x} A_{x}}\left|\left(\langle\varphi\rangle_{\langle\psi\rangle}+\langle\theta\rangle c_{\langle\psi\rangle}\right)\right|}\left\{1+\frac{\varphi^{\prime} s_{\langle\psi\rangle}+\theta^{\prime} c_{\langle\psi\rangle}}{2\left(\langle\varphi\rangle s_{\langle\psi\rangle}+\langle\theta\rangle c_{\langle\psi\rangle}\right\rangle}\right\}, \\
\widehat{w}_{y}=-\operatorname{sgn}\left(-\langle\varphi\rangle_{\langle\psi\rangle}+\langle\theta\rangle_{\langle\psi\rangle}\right) \sqrt{\frac{2 m g}{\rho c_{x} A_{x}} \mid\left(-\left\langle\varphi c_{\langle\psi\rangle}+\langle\theta\rangle_{\langle\psi\rangle}\right) \mid\right.}\left\{1+\frac{-\varphi^{\prime} c_{\langle\psi\rangle}+\theta^{\prime} s_{\langle\psi\rangle}}{2\left(-\langle\varphi\rangle c_{\langle\psi\rangle}+\langle\theta\rangle_{\langle\psi\rangle}\right\rangle}\right\}
\end{gathered}
$$

in the square-law case. Equations (22)-(25) imply that the estimates of the horizontal components of the wind velocity are the sums of the regular and fluctuation parts regardless of the drag model. The regular part of the estimates is determined by the average values of the Euler angles, and the fluctuation part is proportional to the fluctuations of the roll and pitch angles. Thus, equations (22)(25) allows us estimate the longitudinal and transverse components of the wind velocity fluctuations. 


\section{Estimates of longitudinal and transverse components}

Let us define estimates the longitudinal and transverse components of wind field fluctuations by analogy with Eqs. (9) and (10) as

$$
\begin{gathered}
\widehat{w}_{r}^{\prime}(\boldsymbol{r}, t)=\hat{n}_{x} \widehat{w}_{x}^{\prime}(\boldsymbol{r}, t)+\hat{n}_{y} \widehat{w}_{y}^{\prime}(\boldsymbol{r}, t), \\
\widehat{w}_{t}^{\prime}(\boldsymbol{r}, t)=-\hat{n}_{x} \widehat{w}_{y}^{\prime}(\boldsymbol{r}, t)+\hat{n}_{y} \widehat{w}_{x}^{\prime}(\boldsymbol{r}, t),
\end{gathered}
$$

where $\widehat{w}_{r}^{\prime}(\boldsymbol{r}, t)$ is the estimate of the longitudinal component, and $\widehat{w}_{t}^{\prime}(\boldsymbol{r}, t)$, of the transverse component in the XoY plane. The estimate of the vector direction $\widehat{\boldsymbol{n}}$ in Eqs. (26) and (27) can be written as

$$
\widehat{\boldsymbol{n}}=\left\{\hat{n}_{x}, \hat{n}_{y}, 0\right\}=\left\{\frac{\left\langle\hat{w}_{x}\right\rangle}{\left\langle\hat{w}_{\|}\right\rangle}, \frac{\left\langle\hat{w}_{y}\right\rangle}{\left\langle\hat{w}_{\|}\right\rangle}, 0\right\},
$$

where $\left\langle\widehat{w}_{x}\right\rangle$ and $\left\langle\widehat{w}_{y}\right\rangle$ are the estimates of the components of the horizontal wind velocity along the $\mathrm{x}$ and $\mathrm{y}$ axes; $\widehat{w}_{\|}=\sqrt{\left\langle\widehat{w}_{x}\right\rangle^{2}+\left\langle\widehat{w}_{y}\right\rangle^{2}}$ is the estimate of the horizontal wind velocity.

It follows from Eqs. (26) and (27) that the estimates of the longitudinal and transverse components could be used for calculation of fluctuation spectra of the its components using quadcopter data. AMK-03 is the $3 \mathrm{D}$ acoustic anemometer that measures the longitudinal and transverse components, so Eqs. (9), (10), (26) and (27) allows to calculate the fluctuation spectra and compare them in the turbulent atmosphere.

\section{General information about experiments}

Experiments were carried out in the Geophysical Observatory of IMCES SB RAS on February 20 and August 13, 2020. The Observatory is situated in Tomsk Akademgorodok, on the territory with complex orography, in a parkland zone with buildings of research institutes and motorways.

Figure 1 shows the quadcopter flight trajectory during the experiments. A DJI Phantom 4 Pro drone was launched on February 20, 2020, in close proximity to the Observatory building. After takeoff, it climbed to an altitude of $28 \mathrm{~m}$ and flew up to the AMK-03 automated weather station, which was mounted at a mast on the Observatory roof. The quadcopter axis $\mathrm{x}$ was approximately northward oriented. The experiment started at 10:21 UTC and ended at 10:34 UTC. The quadcopter held the altitude near AMK-03 for about $10 \mathrm{~min}$ and then returned to the starting point.

On August 13, 2020, the drone started near the 30-m meteorological mast situated between the Observatory and a forest. After takeoff, it climbed to an altitude of $27 \mathrm{~m}$ and flew up to AMK-03 automated weather station mounted at the mast. The quadcopter axis $x$ was also approximately northward oriented. The experiment was carried out from 07:01 to 07:22 UTC. The quadcopter held the altitude near AMK-03 for about 18 min and then returned to the starting point.

AMK-03 is designed to measure and record wind speed and direction, air temperature and relative humidity, and atmospheric pressure. We used two AMK-03 stations. The station on the Observatory roof measured wind speed and direction with a frequency of $10 \mathrm{~Hz}$, and the weather station at the 30-m meteorological mast recoded the time series with a frequency of $80 \mathrm{~Hz}$. The quadcopter flight data (time series of the roll, pitch, and yaw angles) were recorded with a frequency of $10 \mathrm{~Hz}$. When calculating the spectra of wind velocity fluctuations, the AMK-03 data were reduced to a frequency of $10 \mathrm{~Hz}$ by means of partial averaging of the time series. 


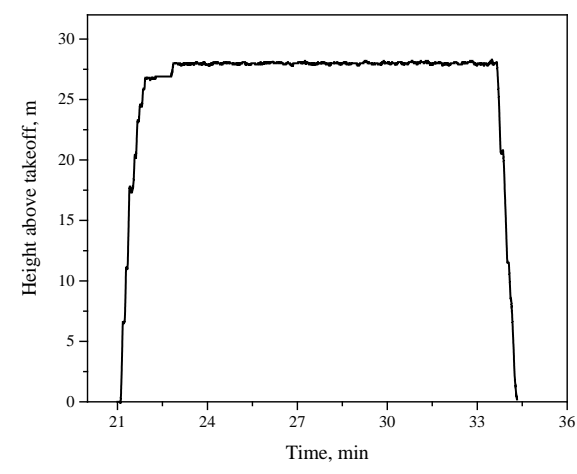

(a)

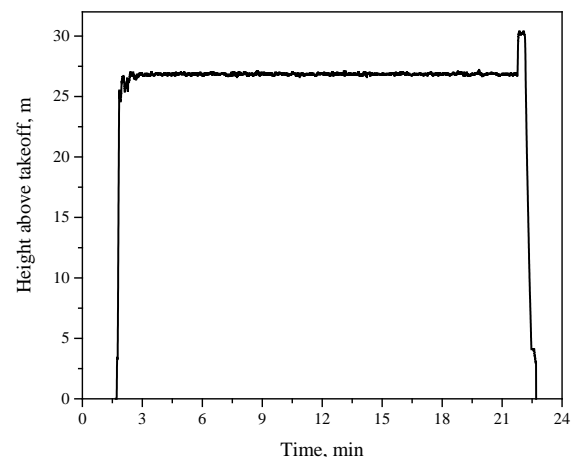

(b)

Figure 1. Quadcopter flight trajectory during the experiments on (a) February 20 and (b) August 13, 2020.

According to data of the Tomsk International Airport, which is located at a distance of $\sim 10 \mathrm{~km}$ from the building of the Geophysical Observatory of IMCES SB RAS, the weather conditions were difficult for quadcopter flight during the experiment on February 20: south-southwestern wind at a speed of $6.0 \mathrm{~m} / \mathrm{s}$, air temperature of $-1.5^{\circ} \mathrm{C}$, air humidity of $100 \%$, and snowing. During the experiment on August 13, the southeastern wind at a speed of $4 \mathrm{~m} / \mathrm{s}$, air temperature of $26^{\circ} \mathrm{C}$, and air humidity of $70 \%$, and no precipitation were recorded.

\section{Measurement results}

Figure 2 shows variations in the quadcopter velocity components along the $\mathrm{x}, \mathrm{y}$, and $\mathrm{z}$ axes during hovering. The quadcopter velocity components were generally zero during the measurements. Sometimes, the forces which acted on the quadcopter exceeded the capabilities of the control system for a short time, and high-precision positioning was perturbed.
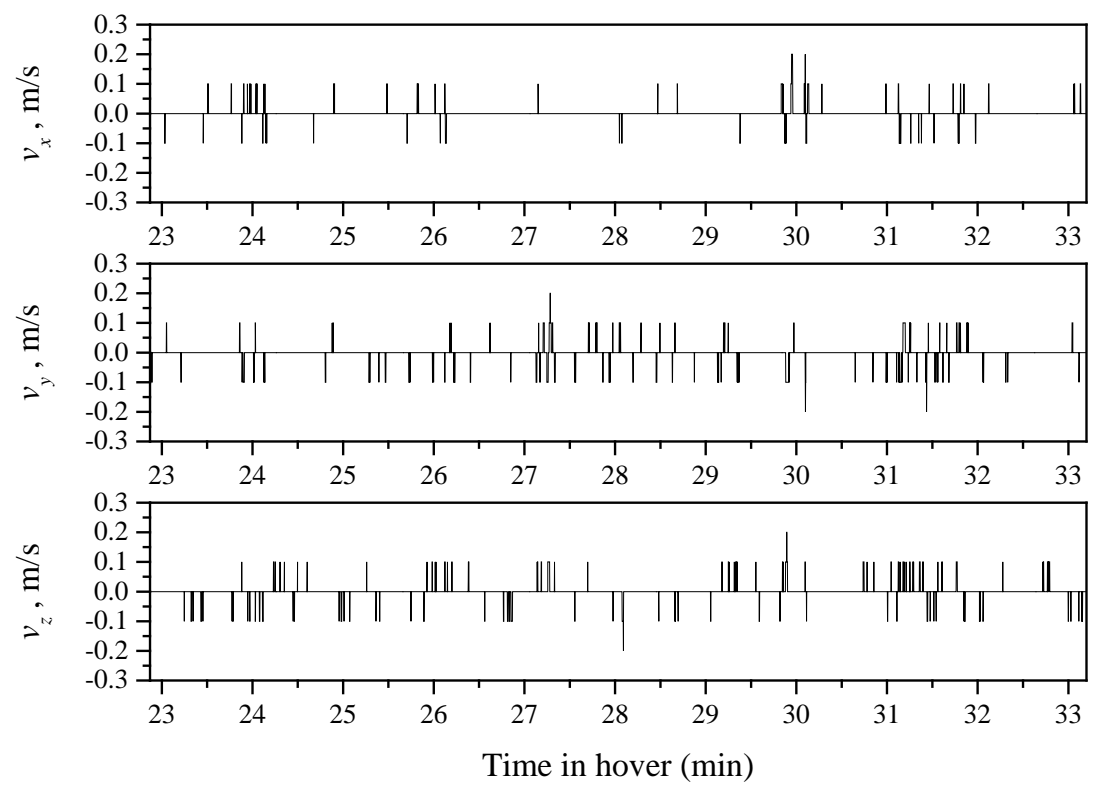

(a) 

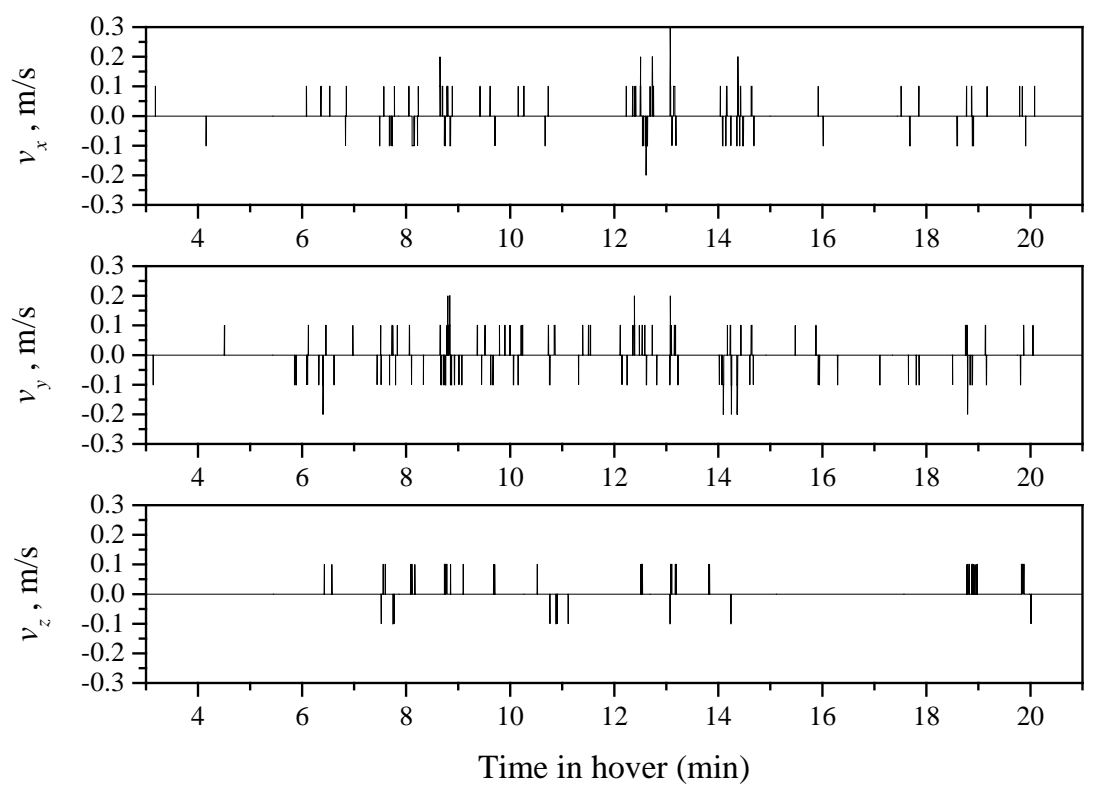

(b)

Figure 2. Quadcopter velocity components along the $x, y$, and $z$ axes during hovering (a) on February 20 and (b) August 13, 2020.

After control recovery, the quadcopter began moving to its original position and stopped upon reaching it. Thus, the time periods when the spatial positioning of the quadcopter was perturbed can be ignored due to their insignificance, and the hover could be considered ideal from 10:23:00 to 10:33:00 UTC on February 20 and 07:01 to 07:22 UTC on August 13, 2020.
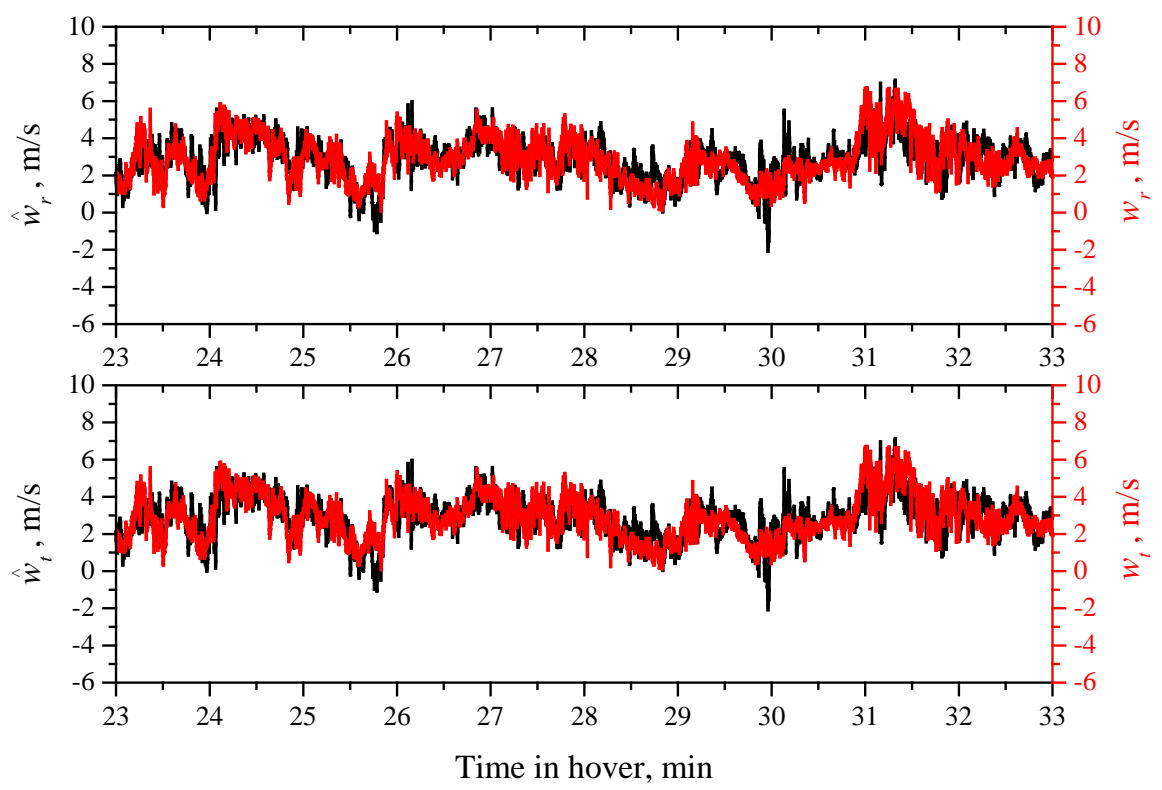

(a) 


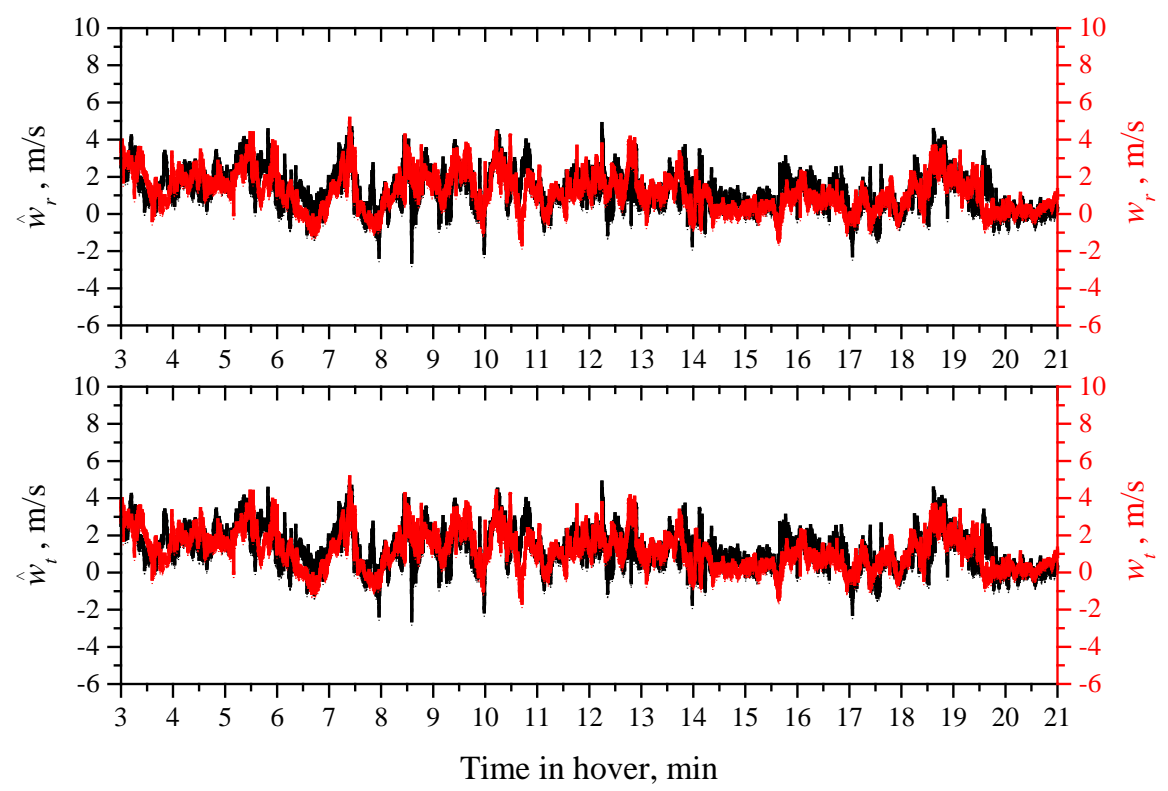

(b)

Figure 3. Temporal variations in the estimates of the longitudinal $\widehat{w}_{r}$ and transverse $\widehat{w}_{t}$ components of the horizontal wind velocity (black curve) and in the longitudinal $w_{r}$ and transverse $w_{t}$ wind velocity components (red curve) (a) on February 20 and (b) August 13, 2020.

Let us consider the behavior of the estimates of the longitudinal and transverse wind velocity components in a turbulent atmosphere in the altitude holding mode. Figure 3 shows the time series of these components (red curve) measured by AMK-03 automated weather station and their estimates (black curve) found on the basis of the DJI Phantom 4 Pro quadcopter data. It follows from Fig. 3 that the time series of $\widehat{w}_{r}$ and $w_{r}$ and the time series of $\widehat{w}_{t}$ and $w_{t}$ generally coincide, and the differences are observed in the region of high-frequency fluctuations.

For objective assessment of the spectra of fluctuations of the wind field components and spectra of their estimates in a turbulent atmosphere, we used averaging over the hover period. The spectra were calculated with the use of standard FFT algorithms and a smoothing procedure. 


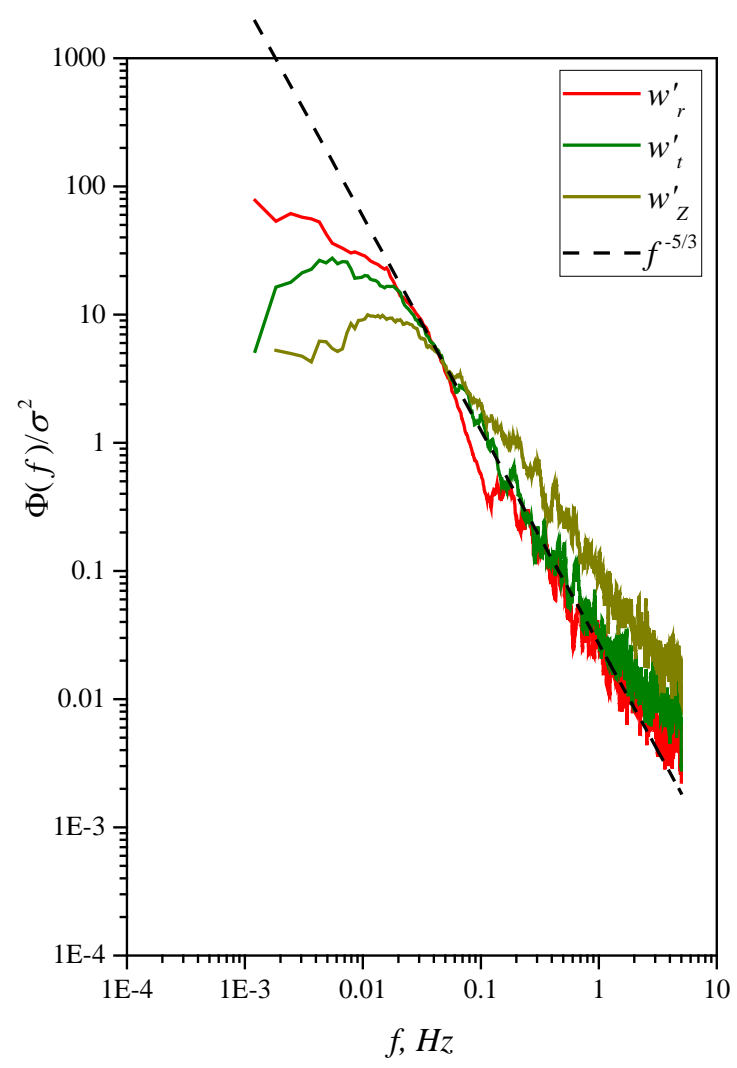

(a)

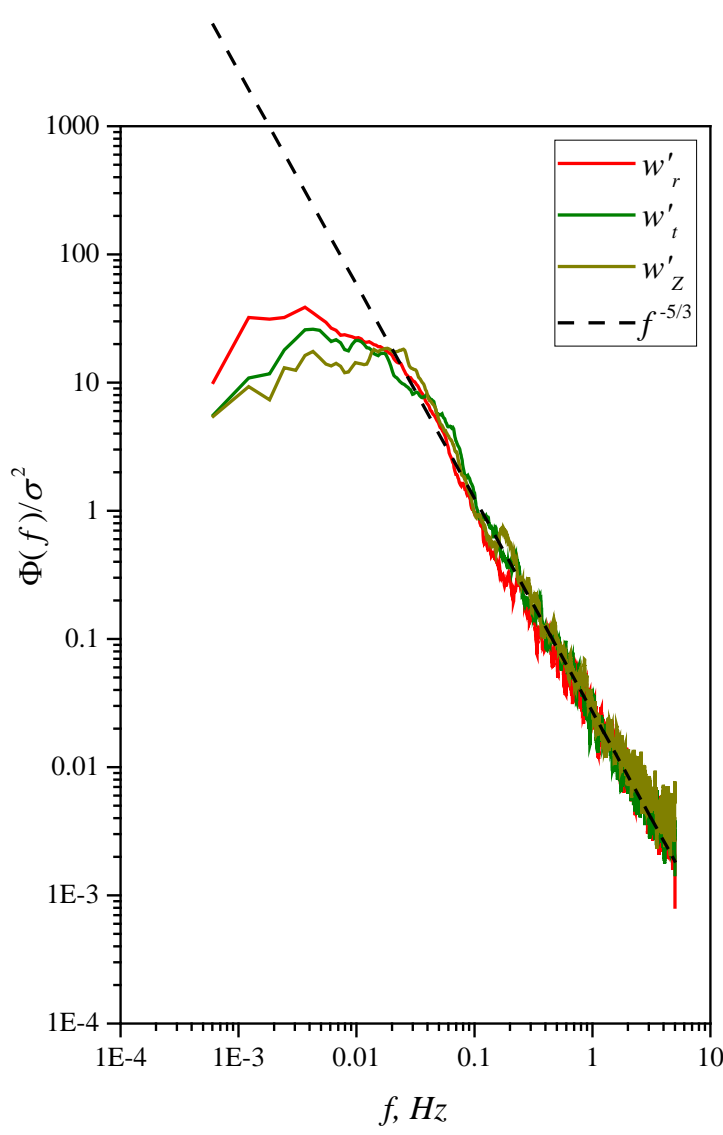

(b)

Figure 4. Spectra of turbulent fluctuations of wind field components (a) on February 20 and (b) August 13, 2020; the dashed line shows the power spectrum of fluctuations $\Phi(f) \sim f^{-5 / 3}$, which corresponds to the Kolmogorov-Obukhov " $-5 / 3$ " law; $\sigma^{2}$ is the normalization coefficient

Figure 4 shows relative fluctuation spectra of the components calculated on the basis of AMK-03 data for two experiments. It is seen from figure that anisotropy of the wind velocity fluctuations was observed on February 20, but on August 13 this phenomenon was not observed: the fluctuation spectra of all components $w_{r}^{\prime} w_{t}^{\prime}$ and $w_{Z}^{\prime}$ coincide. In the inertial interval, the spectra of the $w_{r}^{\prime}, w_{t}^{\prime}$, and $w_{Z}^{\prime}$ components behave similarly to the spectrum which corresponds to the Kolmogorov-Obukhov "-5/3" law.

Figure 5 shows the results of comparison between the fluctuation spectra of the estimates of transverse and longitudinal components. One can see that the fluctuation spectra of the estimates of transverse and longitudinal components $\widehat{w}_{\mathrm{r}}^{\prime}$ and $\widehat{w}_{\mathrm{t}}^{\prime}$ coincide in the altitude holding mode. In the inertial range, the spectra of the $\widehat{w}_{\mathrm{r}}^{\prime}$ and $\widehat{w}_{\mathrm{t}}^{\prime}$ components coincide with the spectrum which corresponds to the Kolmogorov-Obukhov "-5/3" law. 


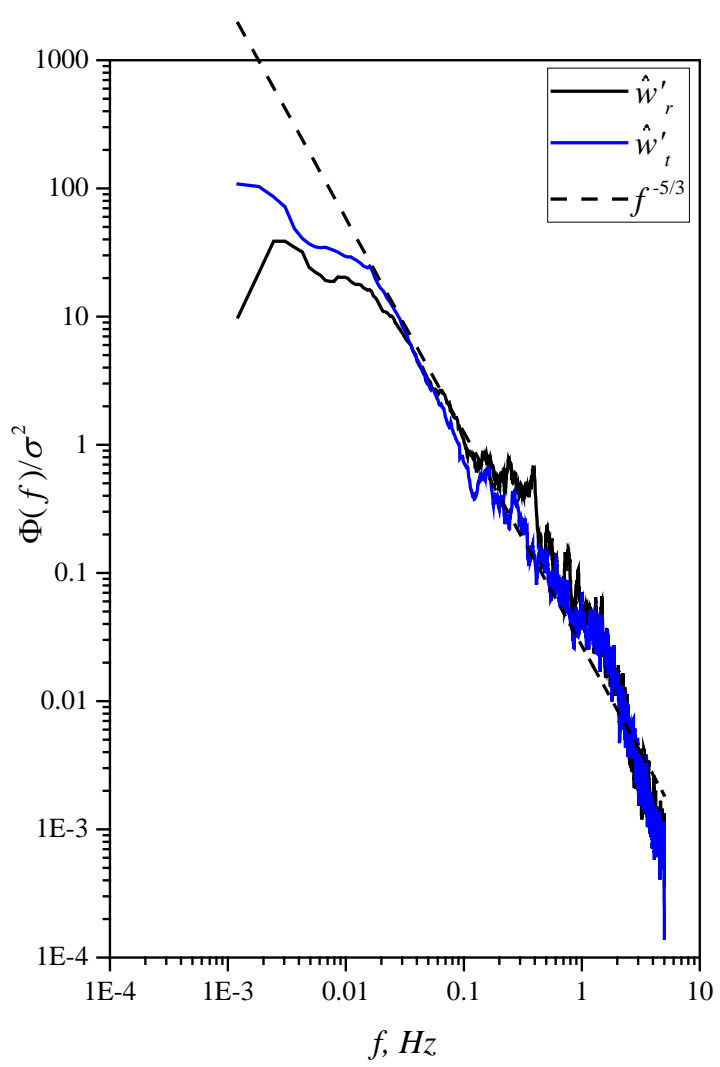

(a).

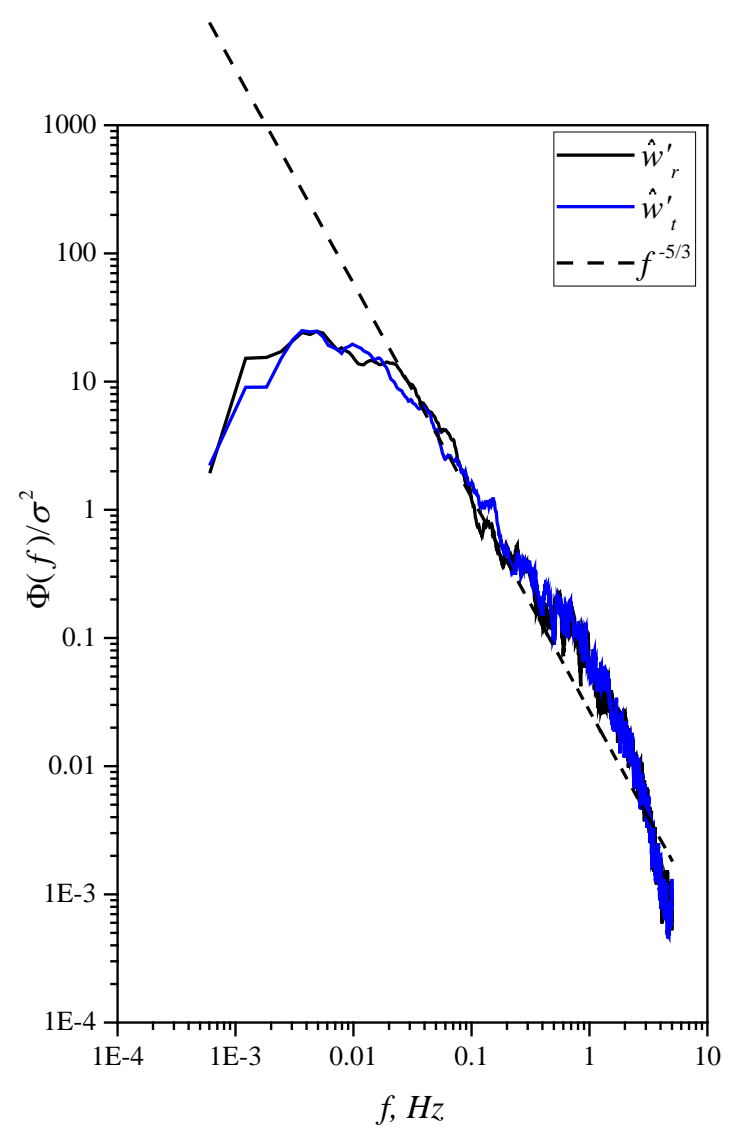

(b)

Figure 5. Spectra of turbulent fluctuations of the longitudinal and transverse components of the wind field (a) on February 20 and (b) August 13, 2020.

Figure 5 shows the results of comparison between the fluctuation spectra of the estimates of transverse and longitudinal components. One can see that the fluctuation spectra of the estimates of transverse and longitudinal components $\widehat{w}_{\mathrm{r}}^{\prime}$ and $\widehat{w}_{\mathrm{t}}^{\prime}$ coincide in the altitude holding mode. In the inertial range, the spectra of the $\widehat{w}_{r}^{\prime}$ and $\widehat{w}_{t}^{\prime}$ components coincide with the spectrum which corresponds to the Kolmogorov-Obukhov "-5/3" law. 


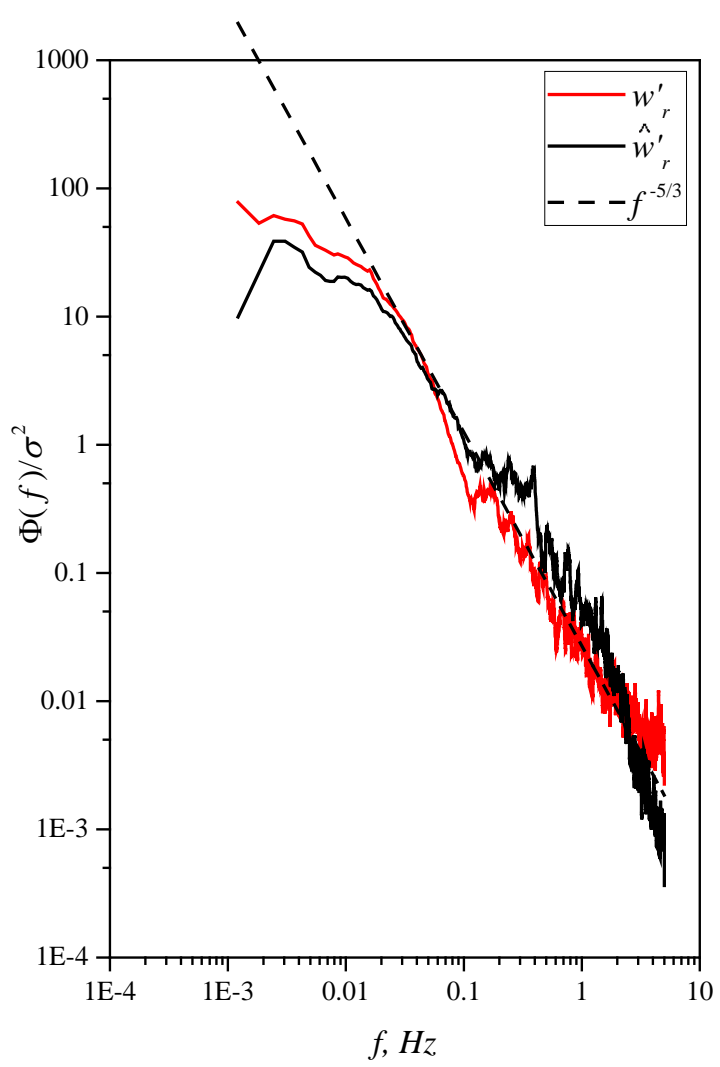

(a)

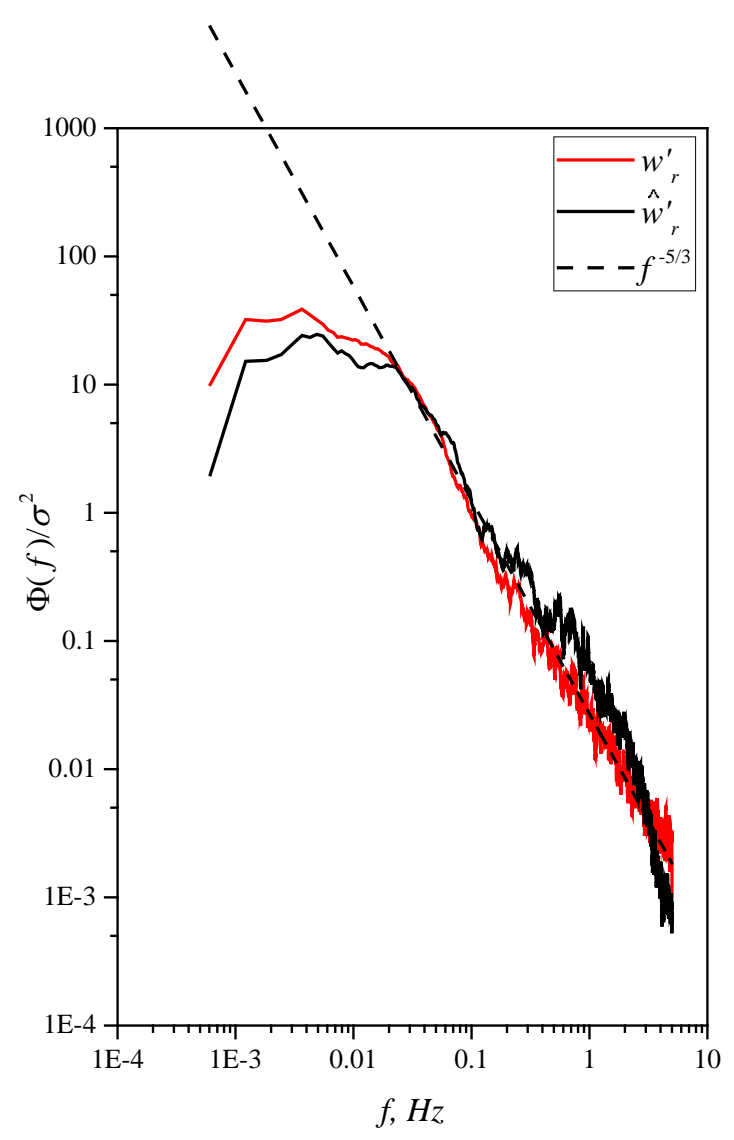

(b)

Figure 6. Spectra of turbulent fluctuations of the longitudinal wind velocity component $w_{r}^{\prime}$ and of its estimate $\widehat{w}_{r}^{\prime}$ (a) on February 20 and (b) August 13, 2020.

Figures 6 and 7 show the results of comparison between the fluctuation spectra of the transverse and longitudinal components and of their estimates. The fluctuation spectra of $w_{r}^{\prime}$ and $\widehat{w}_{r}^{\prime}$ and $w_{t}^{\prime}$ and $\widehat{w}_{t}^{\prime}$ generally coincide, and significant differences are seen in the high-frequency spectral region. Here, the frequency spectra of turbulent fluctuations of $\widehat{w}_{r}^{\prime}$ and $\widehat{w}_{t}^{\prime}$ damp with the frequency faster that according to the Kolmogorov-Obukhov "-5/3" law. 


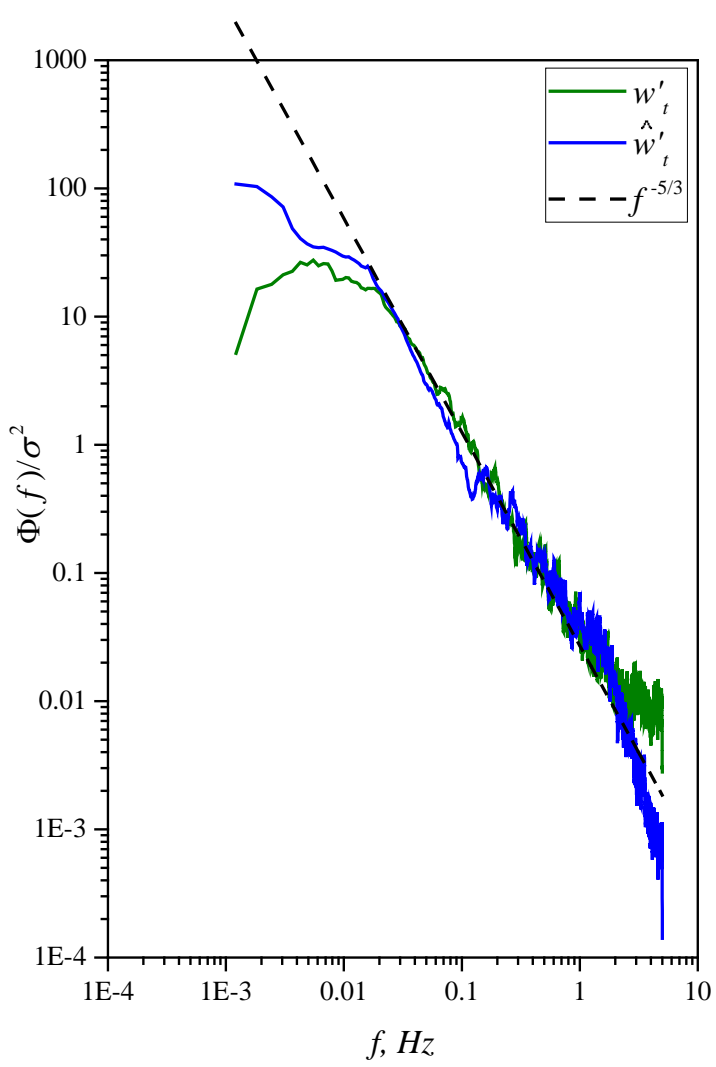

(a)

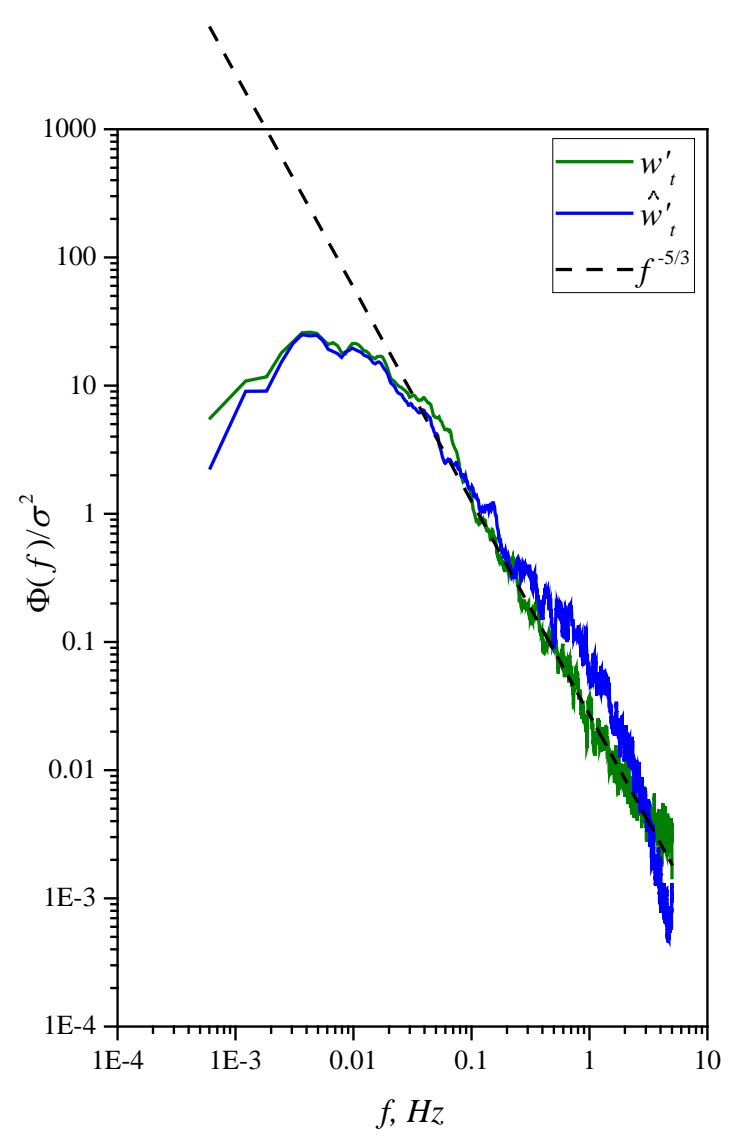

(b)

Figure 7. Spectra of turbulent fluctuations of the transverse wind velocity component $w_{t}^{\prime}$ and its estimate $\widehat{w}_{t}^{\prime}$ (a) on February 20 and (b) August 13, 2020.

\section{Discussion}

Based on the study of the spectra of turbulent fluctuations of the longitudinal and transverse wind velocity components, measured with the automated weather station AMK-03 and DJI Phantom 4 Pro quadcopter, the following conclusions can be drawn.

We used DJI Phantom 4 Pro quadcopter in our experiments, for which we have shown that the fluctuations spectra of the transverse and longitudinal components measured with the 3D acoustic anemometer and an UAV generally coincide in a wide spectral range. In the inertial range, the behavior of the spectra coincides with the spectrum, which corresponds to the KolmogorovObukhov " $-5 / 3$ " law. The fluctuations spectra of these components measured with the 3D acoustic anemometer and an UAV coincide regardless of the state of the turbulent atmosphere: for isotropic and anisotropic turbulence. This means that the state of a turbulent atmosphere can be controlled with the use of a rotary-wing UAV instead of contact sensors, e.g., 3D acoustic anemometer.

The low-altitude sounding of a turbulent atmosphere with the use of a fixed-wing UAV [8-15] allow retrieving information from spatially averaged data. However, for certain scientific and applied problems, for example, traffic control of UAV of "smart city", detailed information is required on local processes which run in a turbulent atmosphere. For such problems, rotary-wing UAV capable of hovering at different altitudes for a long time have an undoubted advantage.

Differences in the spectra of turbulent fluctuations obtained with AMK-03 and DJI Phantom 4 Pro are observed in the high-frequency spectral region, which corresponds to a small-scale low-energy turbulence. One possible reason for the difference in the spectra is the presence of an 
induced turbulence: in the hovering mode, the quadcopter creates high-energy turbulent vortices around it, which destroy the small-scale structure of atmospheric turbulence. As a result, the measured spectrum of turbulent fluctuations of the wind field in the high-frequency spectral region may differ from the spectrum which exists in the atmosphere.

Another possible reason could be large mass and size of the quadcopter used. A small-scale turbulence has low kinetic energy; hence, the quadcopter is insensitive to weak high-frequency impacts due to high inertia and large size. It is possible that a lighter and smaller quadcopter will be more sensitive to small-scale turbulent fluctuations and will produce weaker local turbulent distortions, which is to provide a correct pattern in the high-frequency spectral region. Therefore, the future direction of our research is to study the effect of atmospheric turbulence on the operation of a quadcopter with lower mass and smaller size than the DJI Phantom 4 Pro.

Author Contributions: Conceptualization, AS; methodology, AS; software, AA, ES; validation, AK, AT and $\mathrm{AM}$; investigation, $\mathrm{AS}, \mathrm{AA}, \mathrm{AK}$; data curation, $\mathrm{AK}$; writing-original draft preparation, OP; writing-review and editing, AS. supervision, AS; project administration, AS.

Funding: The reported study was funded by RFBR, project number 19-29-06066 mk.

\section{References}

1. Stith, J.L.; Baumgardner, D.; Haggerty, J.; Hardesty, M.; Lee, W.; Lenschow, D.; Pilewskie, P.; Smith, P.L.; Steiner, M.; Vömel, H. 100 Years of Progress in Atmospheric Observing Systems Meteorological Monographs 2018, 59 .

2. Palomaki, R.T.; Rose, N.T.; van den Bossche, M.; Sherman, T.J.; De Wekker, S.F.J. Wind estimation in the lower atmosphere using multirotor aircraft J. Atmos. Ocean. Technol. 2017, 34, 1183-1190.

3. González-Rocha, J.; De Wekker, S.F.J.; Ross, S.D.; Woolsey, C.A. Wind Profiling in the lower atmosphere from wind-induced perturbations to multirotor UAS Sensors 2020, 20, 1341.

4. González-Rocha, J. and Woolsey, C. A., Cornel Sultan measuring atmospheric winds from quadrotor motion AIAA Atmospheric Flight Mechanics Conference, Grapevine, Texas, USA, 2017.

5. Cornman, L.B.; Chan, W.N. Summary of a Workshop on Integrating Weather into Unmanned Aerial System Traffic Management Bull. Amer. Meteor. Soc. 2017, 98(10), ES257-ES259.

6. Wang, B.H.; Wang, D.B.; Ali, Z.A.; Ting, B.T.; Wang, H. An overview of various kinds of wind effects on unmanned aerial vehicle Measurement and Control. 2019, 52(7-8), 731-739.

7. Varentsov, M.I.; Artamonov, A.Yu.; Pashkin, A.D.; Repina, I.A. Experience in the quadcopter-based meteorological observations in the atmospheric boundary layer. IOP Conf. Ser.: Earth Environ. Sci. 2019, $231,012053$.

8. Reuder, J.; Brisset, P.; Jonassen, M.; Muller, M.; Mayer, S. The Small Unmanned Meteorological Observer SUMO: A new tool for atmospheric boundary layer research. Meteorologische Zeitschrift 2009, 18(2), $141-$ 147.

9. Reuder, J.; Jonassen, M.O. First results of turbulence measurements in a wind park with the Small Unmanned Meteorological Observer SUMO Energy Procedia 2012, 24, 176-185.

10. Reuder, J.; Jonassen, M.O. Proof of concept for wind turbine wake investigations with the RPAS SUMO Energy Procedia 2016, 94, 452 - 461.

11. Reineman, B.D. Development and Testing of Instrumentation for UAV-Based Flux Measurements within Terrestrial and Marine Atmospheric Boundary Layers Journal of Atmospheric and Oceanic Technology 2013, 30, 1295-1319.

12. Reineman, B.D.; Lenain, L.; Melville, W.K. The Use of Ship-Launched Fixed-Wing UAVs for Measuring the Marine Atmospheric Boundary Layer and Ocean Surface Processes Journal of Atmospheric and Oceanic Technology 2016, 33, 2029-2052.

13. Balsley, B.B.; Lawrence, D.A.; Fritts, D.C.; Wang, L.; Wan, K.; Werne, J. Fine Structure, Instabilities, and Turbulence in the Lower Atmosphere: High-Resolution In Situ Slant-Path Measurements with the DataHawk UAV and Comparisons with Numerical Modeling Journal of Atmospheric and Oceanic Technology 2018, 35, 619-642.

14. Rautenberg, A.; Graf, M.; Wildmann, N.; Platis, A.; Bange, J. Reviewing Wind Measurement Approaches for Fixed-Wing Unmanned Aircraft. Atmosphere 2018, 9, 422. 
15. Rautenberg, A.; Allgeier, J.; Jung, S.; Bange, J. Calibration Procedure and Accuracy of Wind and Turbulence Measurements with Five-Hole Probes on Fixed-Wing Unmanned Aircraft in the Atmospheric Boundary Layer and Wind Turbine Wakes Atmosphere 2019, 10, 124.

16. Shimura, T.; Inoue, M.; Tsujimoto, H.; Sasaki, K.; Iguchi, M. Estimation of Wind Vector Profile Using a Hexarotor Unmanned Aerial Vehicle and Its Application to Meteorological Observation up to $1000 \mathrm{~m}$ above Surface. Journal of Atmospheric and Oceanic Technology 2018, 35, 1621-1631.

17. Neumann, P.P.; Bartholmai, M. Real-time wind estimation on a micro unmanned aerial vehicle using its internal measurement unit Sens. Actuators 2015, 235A, 300-310.

18. Allison, S.; Bai, H.; Jayaraman, B. Wind estimation using quadcopter motion: A machine learning approach Aerosp. Sci. Technol. 2020, 98, 105699.

19. Wang, L.; Misra, G.; Bai, X.; A K Nearest Neighborhood-Based Wind Estimation for Rotary-Wing VTOL UAVs Drones 2019, 3(2), 31.

20. Fuertes, F.C.; Wilhelm, L.; Porté-Agel, F. Multirotor UAV-Based Platform for the Measurement of Atmospheric Turbulence: Validation and Signature Detection of Tip Vortices of Wind Turbine Blades. J. Atmos. Oceanic Technol. 2019, 36(6), 941-955.

21. Simma, M.; Mjøen, H.; Boström T. Measuring Wind Speed Using the Internal Stabilization System of a Quadrotor Drone Drones, 2020, 4, 23.

22. Kim M.; Kwon, B.H. Estimation of Sensible Heat Flux and Atmospheric Boundary Layer Height Using an Unmanned Aerial Vehicle Atmosphere 2019, 10, 363.

23. Azbukin, A.A.; Bogushevich, A.Ya.; Korolkov, V.A.; Tikhomirov, A.A.; Shelevoi, V.D. A field version of the AMK-03 automated ultrasonic meteorological complex Russian Meteorology and Hydrology 2009, 34(2), 133-136.

24. Azbukin, A.A.; Bogushevich, A.Ya.; Kobzev, A.A.; et al. AMK-03 Automatic weather stations, their modifications and applications Sensors and systems 2012, 3(154), 47-52.

25. Monin, A. S.; Yaglom, A. M. Statistical Hydromechanics. Part 2. Turbulent Mechanics, Nauka, Moscow, 1967.

26. Stull, R.B. An Introduction to Boundary Layer Meteorology, Kluwer Academic Publishers, Netherlands 1989.

27. Pounds, P.; Mahony, R.; Corke, P. Modelling and control of a large quadrotor robot Control Engineering Practice, 2010, 18, 691 - 699.

28. Mahony, R.; Kumar, V.; Corke, P. Multirotor aerial vehicles: Modeling, estimation, and control of quadrotor IEEE Robotics Automation Magazine 2012, 19, 20-32,.

29. Michael, N.; Mellinger, D.; Lindsey, Q.; Kumar, V. The grasp multiple micro-UAV testbed IEEE Robotics Automation Magazine, 2010, 17, 56-65.

30. Wang, J.Y.; Luo, B.; Zeng, M.; Meng, Q. A Wind Estimation Method with an Unmanned Rotorcraft for Environmental Monitoring Tasks Sensors 2018, 18(12), 4504 